\title{
Hyaluronan and TLR4 promote surfactant protein C-positive alveolar progenitor cell renewal and prevent severe pulmonary fibrosis in mice
}

\author{
Jiurong Liang ${ }^{1}$, Yanli Zhang ${ }^{1,3}$, Ting Xie ${ }^{1}$, Ningshan Liu ${ }^{1}$, Huaiyong Chen ${ }^{2,3}$, Yan Geng ${ }^{1}$, \\ Adrianne Kurkciyan ${ }^{1}$, Jessica Monterrosa Mena ${ }^{1}$, Barry R. Stripp ${ }^{1}$, Dianhua Jiang ${ }^{1,{ }^{*}}$, and \\ Paul W. Noble ${ }^{1, *}$ \\ 1'Department of Medicine and Women's Guild Lung Institute, Cedars-Sinai Medical Center, Los \\ Angeles, California, USA \\ ${ }^{2}$ Department of Medicine, Duke University School of Medicine, Durham, North Carolina, USA
}

\section{SUMMARY}

Successful recovery from lung injury requires the repair and regeneration of alveolar epithelial cells in order to restore the integrity of gas exchanging regions within the lung and preserve organ function. Improper regeneration of the alveolar epithelium is often associated with severe pulmonary fibrosis, the latter of which involves the recruitment and activation of fibroblasts, as well as matrix accumulation. Type 2 alveolar epithelial cells (AEC2s) are stem cells in the adult lung that contribute to the lung repair process. The mechanisms that regulate AEC2 cell renewal are incompletely understood. We provide evidence that expression of the innate immune receptor Toll like receptor 4 (TLR4) and the extracellular matrix glycosaminoglycan hyaluronan (HA) on AEC2s are important for AEC2 renewal, repair of lung injury, and limiting the extent of fibrosis. Either deletion of TLR4 or HA synthase 2 in surfactant protein-C-positive AEC2s leads to impaired renewal capacity, severe fibrosis and mortality. Furthermore, AEC2s from patients with severe pulmonary fibrosis have reduced cell surface HA and impaired renewal capacity, suggesting that HA and TLR4 are key contributors to lung stem cell renewal and that severe pulmonary fibrosis is the result of distal epithelial stem cell failure.

\footnotetext{
*Correspondence should be addressed to P.W.N. (paul.noble@cshs.org) or D.J. (dianhua.jiang@cshs.org).

${ }^{3}$ Present address: Department of Biochemistry and Molecular Biology, Peking Union Medical College, Tsinghua University, Beijing, China (Y.Z.). Tianjin Haihe Hospital and Tianjin Institute of Respiratory Diseases, Tianjin, China (H.C.).

Accession codes. Gene Expression Omnibus (GEO) repository: microarray data have been deposited with accession number GSE68704.

Note: Supplementary Information and Source Data files are available in the online version of the paper. AUTHORS CONTRIBUTIONS

All authors participated in the design, execution, and interpretation of the study. P.W.N., D.J., and J.L. conceived and designed the study. J.L. performed the majority of experiments and analyzed data. Y.L., T.X., N.L., H.C., Y.G., A.K., J.M.M., and D.J. performed experiments and analyzed data. B.R.S. contributed to the design and interpretation of the study, and provided critical reagents. J.L., D.J., and P.W.N. wrote the manuscript, and P.W.N. and D.J. approved the submission.
}

COMPETING FINANCIAL INTERESTS

The authors declare no competing financial interests. 


\section{INTRODUCTION}

The lung, along with the skin and gut, are the three organs in perpetual contact with the external environment. The gut has evolved mechanisms for mucosal surface protection and repair largely through its co-habitation with commensal bacterial flora. This symbiotic relationship both protects the gut from insult ${ }^{1,2}$ and initiates processes to rapidly invigorate stem cell renewal ${ }^{3}$. In the lung, the alveolar epithelium is vulnerable to noxious injury, and also encompasses a critical stem cell/progenitor niche that can overcome such assaults ${ }^{4,5}$. Timely repair of lung injury is essential for proper restoration of function and determines the outcome of life or death. Inadequate repair can result in abrupt respiratory decompensation and if protracted, potentially fatal outcomes such as fibrosis. During development, type 1 alveolar epithelial cells (AEC1s) and AEC2s arise from a bipotent progenitor cell lineage ${ }^{6}$, whereas after birth, AEC2s can undergo long-term self-renewal and give rise to AEC1s during homeostasis ${ }^{6,7}$, but also in response to infection ${ }^{8,9}$ or tissue damage ${ }^{4,10}$.

The mechanisms that both constitutively protect the distal alveolar space and promote renewal of injured AEC2s are incompletely understood. A critical barrier to progress in fibrosis has been the lack of understanding of the interactions between endogenous host factors generated during non-infectious injury and cellular processes that regulate tissue remodeling. We have shown that the innate immune receptors TLR2 and 4 and the extracellular matrix glycosaminoglycan hyaluronan (HA) play important roles in lung injury and repair processes ${ }^{1-14}$. HA is a glycosaminoglycan polymer comprised of the repeating disaccharide units $N$-acetyl-glucosamine and $D$-glucuronic acid ${ }^{13,15}$ and is synthesized by membrane-bound synthases ${ }^{15}$. Among three HA synthases, HAS2 is most critical for development, since targeted deletion results in an embryonic lethal phenotype due to failed heart and blood vessel development ${ }^{16}$.

Idiopathic pulmonary fibrosis (IPF) is a lethal disease characterized by abnormal lung epithelial cells and overwhelming matrix accumulation in the lung interstitium. Most patients with IPF deteriorate with a median survival of less than 3 years from diagnosis ${ }^{17}$. Although the exact molecular mechanisms of progressive lung fibrosis are unclear, it is believed prevailing hypotheses maintain that IPF results from numerous micro-injuries to the alveolar epithelia that promote excessive, sustained fibroblast activation into matrixproducing myofibroblasts ${ }^{18,19}$. Further, surfactant protein deficiency ${ }^{20}$ or mutations in the surfactant protein C (SFTPC) and SFTPA gene ${ }^{21,22}$ were noted in familial interstitial lung diseases. And a recent study also showed that telomere dysfunction caused alveolar stem cell failure $^{23}$. Collectively, these observations point to alveolar epithelial cells as central in the pathogenesis of IPF, but the renewal capacity of AEC2s from IPF lungs has not been investigated.

In this study, we propose that $\mathrm{HA}$ on the surface of AEC2s functions as a renewal signal. We found that mice with a targeted deletion of Has 2 in surfactant protein C-positive AEC2s or lacking the cognate receptor $T I r 4$, were markedly more susceptible to bleomycin-induced lung injury, developed fulminant interstitial fibrosis, and AEC2 from these mice had impaired renewal capacity compared to wild type (WT) AEC2s. In addition, we found that IL-6 production was diminished in Tlr 4 and Has 2 deficient mice, and that this cytokine 
promoted 3-D co-culture organoid development and reversed the fibrotic phenotype when restored in the early window following lung injury. Furthermore, we observed that AEC2s from patients with IPF were markedly diminished in number, exhibited greatly reduced cell surface HA expression, and impaired renewal capacity compared to AEC2s from lungs of healthy individuals. Collectively, these data suggest that HA and TLR4 appear to promote alveolar stem cell renewal and could lead to novel therapeutic approaches to treat fibrotic lung diseases.

\section{RESULTS}

\section{$\mathrm{TIr}^{-/-}$mice are more susceptible to bleomycin injury}

We previously demonstrated that cell surface HA and TLR2 and 4 on epithelial cells was necessary to sustain basal NF- $\kappa B$ activation and prevent epithelial cell apoptosis ${ }^{11}$. In the current study, we sought to determine if TLR-HA interactions could provide lung epithelial cells with signals to promote renewal in addition to preventing apoptosis. TIr 4 expression was higher in lungs of wild type (WT) C57Bl/6 mice after bleomycin-induced injury compared to uninjured controls (Fig. 1a). Also, as compared to WT mice $T l r 4^{-/}$mice were more susceptible to bleomycin-induced lung injury (Fig. 1b), and they demonstrated a markedly enhanced fibrotic response to even low doses of bleomycin as illustrated by enhanced trichrome staining (Fig. 1c) and higher hydroxyproline content in lung tissues 21 days after bleomycin (Fig. 1d). More severe fibrosis in the mutant mice was also accompanied by higher a smooth muscle actin (a-SMA) staining (Fig. 1e) and elevated Acta2 (encoding a-SMA) expression (Fig. 1f) as compared to WT mice. The more fibrotic phenotype seemed to be specific to TLR4 deficiency since the enhanced susceptibility to fibrosis was not observed with $T / r 2^{-/-}$mice (Supplementary Fig. 1a,b).

We investigated the inflammatory response of $T 14^{-/}$mice to bleomycin lung injury and found that the number of total bronchoalveolar lavage fluid (BALF) cells as well as differential inflammatory cell counts were not different between $\mathrm{Tlr}^{-/-}$and WT mice (Supplementary Fig. 2a). Further, CXCL2 (MIP-2) in the BALF of $T 1 \mathrm{r}^{-/-}$mice was lower at day 1 after bleomycin compared to WT (Supplementary Fig. 2b). Also, there was no difference in TGF- $\beta$ concentrations in the BALF between $T l r 4^{-/-}$and WT mice (Supplementary Fig. 2c).

\section{Worse alveolar epithelial cell injury in $\mathrm{TIr}^{-/-}$mice}

Flow cytometry analysis showed that AEC2s abundantly expressed TLR4 (Supplementary Fig. 3a). Macrophages also expressed TLR4 as expected and fibroblasts demonstrated minimal TLR4 expression (Supplementary Fig. 3a). In contrast, TLR2 expression on AEC2s was minimal (Supplementary Fig. 3b). Cell surface HA on AEC2s from $\mathrm{Tlr}^{-/-}$was as abundant as that of the WT AEC2s, while the cell surface HA was markedly lower on the Tlr $4^{-1}$ AEC2s (Supplementary Fig. 3c).

We used a mouse lung tissue dissociation and fractionation approach to generate an epithelial cell population (R3, CD45- CD31-CD34-EpCAM ${ }^{+}$; Supplementary Fig. 3d) from which an enriched $\mathrm{AEC} 2$ fraction was isolated by selection of the $\mathrm{CD}^{2} 4^{-} \mathrm{Sca} 1^{-}$subset (R4; 
designated CD24-Sca-1- AEC2s hereafter, Supplementary Fig. 3d) ${ }^{24}$. We verified that the gated CD24-Sca-1- AEC2s were SFTPC ${ }^{+}$AEC2s by analyzing lung cells isolated from mice harboring a SFTPC-GFP transgene (Supplementary Fig. 3e,f) or lineage tracing of AEC2s in SFTPC-CreER;Rosa26-Tomato mice (Supplementary Fig. 3g,h). In uninjured SFTPC-GFP transgenic mouse lungs, $96.1 \%$ of CD24-Sca-1- AEC2s were GFP positive (Supplementary Fig. 3e), and 90.6\% $\mathrm{GFP}^{+}$cells sorted into the CD24-Sca-1- gate (Supplementary Fig. 3f). Similarly, in the lung of uninjured SFTPC-CreER;Rosa26-Tomato mice after four tamoxifen injections, $90.8 \%$ CD24-Sca- $1^{-}$AEC2s were Tomato $^{+}$cells (Supplementary Fig. 3g), and $87.5 \%$ of lineage labeled Tomato ${ }^{+}$cells sorted into the CD24-Sca-1- AEC2 gate (Supplementary Fig. 3h). Immunofluorescence staining showed 96.67\% of FACS-enriched CD24-Sca-1- ${ }^{-}$AEC2s were SP-C ${ }^{+}$(Supplementary Fig. 3i).

Bleomycin exposure is associated with both a lower percentage of $\mathrm{CD} 24^{-} \mathrm{Sca}-1^{-} \mathrm{AEC} 2 \mathrm{~s}$ within the lung epithelial cell fraction (Fig. 2a) and a lower absolute number of CD24-Sca-1- ${ }^{-}$AEC2s recovered from dissociated lung tissue (Fig. 2b) compared to day 0. The nadir in the lower percentage of CD24-Sca-1- AEC2s was observed at day 7 (Fig. 2a) and the fewest number of $\mathrm{CD} 24^{-} \mathrm{Sca}-1^{-}$AEC2s were recovered from lungs at day 3 (Fig. $2 \mathrm{~b}$ ) after bleomycin treatment. We reasoned that the decline in percentage of AEC2s and absolute number were not identical due to other cell types in the lung being differentially impacted by injury. The percentage and number of epithelial cells gradually recovered between day 14 and day 21 after bleomycin (Fig. 2a,b). Tlr4 expression levels of CD24-Sca-1- AEC2s were lower at day 3 and then at equivalent levels by day 14 after bleomycin injury compared to that of AEC2s from uninjured lungs (Fig. 2c). The Tlr4 expression pattern correlated with the pattern of $\mathrm{CD} 24^{-} \mathrm{Sca}-1^{-}$AEC2 damage and recovery after bleomycin injury. Furthermore, we observed even lower levels of CD24-Sca-1- AEC2s in bleomycin injured $\mathrm{TIr}^{-/-}$mouse lungs at multiple time points after injury as compared to injured WT mice (Fig. 2b,d). There was no difference in CD24-Sca-1- AEC2 numbers recovered from uninjured $T I r 4^{-1}$ mouse lungs and WT mouse lungs (Fig. 2b). In addition, we observed that a CD24-Sca- $1^{+}$population emerged following bleomycin lung (Fig. 2a). The significance of these cells in lung injury is under investigation.

\section{AEC2 proliferation and renewal require TLR4 signaling}

To assess the role of TLR4 on AEC2 proliferation in vivo, we performed bromodeoxyuridine (BrdU) labeling after bleomycin treatment. Immunofluorescence staining showed fewer SFTPC and BrdU double positive cells in $T I r 4^{-/}$lungs compared to WT mice 5 days after bleomycin treatment (Fig. 3a,b). Similar fewer cells compared to WT mice were also observed upon Ki67 staining of AEC2s in $\mathrm{Tlr}^{-1-}$ mice (Fig. 3c,d).

We next compared the colony forming efficiency (CFE) of CD24-Sca-1- $\mathrm{AEC} 2 \mathrm{~s}$ isolated from $\mathrm{Tlr} 4^{-/}$and WT mice at day 3 after bleomycin injury. Flow sorted CD24-Sca- $1^{-}$ AEC2s were co-cultured with mouse lung fibroblasts (Mlg 2908) embedded in matrigel medium mix for the generation of clonally-derived 3D organoids. Immunofluorescence staining showed that the epithelial cells at the periphery of organoids were $\mathrm{SFTPC}^{+}$and those within the interior were $\mathrm{PDPN}^{+}(\mathrm{T} 1 \mathrm{a})$ indicative of AEC2 and AEC1 differentiation, respectively (Fig. 3e). Analysis of the replating efficiency of CD24-Sca-1- AEC2s isolated 
from day 3 bleomycin-treated lungs of WT mice revealed sustained regenerative capacity within P1 and P2 cells (Fig. 3f). CD24-Sca-1- AEC2s from day 3 bleomycin injured $\mathrm{Tl}^{-} 4^{-1}$ lungs formed markedly fewer (Fig. 3g) and smaller colonies (Fig. 3h) than did CD24-Sca-1AEC2s cells isolated from WT lungs, suggesting the renewal capacity of $T 1 r 4^{-/-}$AEC2s was impaired. CFEs were similar between AEC2 cells isolated from uninjured $T 1 \mathrm{r}^{-/}$and WT lungs (Supplementary Fig. 4a).

Next we tested the hypothesis that TLR4 promotes AEC2 renewal requires the interaction with HA. Addition of high molecular mass HA (Healon) to the Matrigel medium mix increased CFEs of CD24-Sca-1- ${ }^{-}$AEC2s from day 3 bleomycin-injured WT mice but not TIr $4^{-1}$ AEC2s (Fig. 3i). Healon increased CFEs of CD24-Sca-1- AEC2s from uninjured WT mice, and the HA blocking peptide, pep- $1^{11,25}$, was able to inhibit HA-induced CFEs (Fig. 3j). We investigated the apoptosis of epithelial cells in the bleomycin injured lungs and found that $\mathrm{Tlr}^{-/-}$mice showed more epithelial cell apoptosis at day 3 after bleomycin treatment compared to that of WT mice (Supplementary Fig. 4b).

\section{HA on AEC2s regulates AEC2 renewal and fibrosis}

To further define the role of HA on AEC2 renewal we crossed Has $2^{\text {flox/flox }}$ mice ${ }^{26,27}$ with $S F T P C-C r e$ mice $^{28}$ to generate $S F T P C-C r e ; H a s 2^{\text {flox/flox }}$ mice. The SFTPC-Cre;Has $2^{\text {flox/flox }}$ mice have targeted deletion of Has 2 in distal lung epithelial cells, including AEC2s, throughout lung development ${ }^{7,24}$. The lungs of SFTPC-Cre;Has $2^{\text {flox/flox }}$ mice as well as Has $2^{\text {flox/flox }}$ and SFTPC-Cre transgenic mice developed normally with no readily observable gross or histological abnormalities (Supplementary Fig. 5a,b). The HA concentrations in BALF of uninjured SFTPC-Cre;Has $2^{\text {flox/flox }}$ mice was slightly lower relative to littermates (Supplementary Fig. 5c). There was no difference in the numbers of CD24-Sca-1- AEC2s recovered from uninjured lungs between $S F T P C$-Cre; Has $2^{\text {flox/flox }}$ mice and littermates (Supplementary Fig. 5d). Cell surface HA was markedly lower on CD24-Sca-1- AEC2s isolated from SFTPC-Cre;Has $2^{\text {flox/flox }}$ mice (Fig. 4a). The HA concentrations in the culture medium of CD24-Sca-1- AEC2s from SFTPC-Cre;Has $2^{\text {flox/flox }}$ mice were also lower compared to that of cells from littermates (Fig. 4b).

To determine if lower HA expression impacts the renewal capacity of AEC2s, we isolated CD24-Sca-1- AEC2s from bleomycin injured mouse lungs and recovered far fewer cells from the lungs of SFTPC-Cre;Has $2^{\text {flox/flox }}$ mice compared to littermates (Fig. 4c). Ki67 staining of gated CD24-Sca-1- AEC2s from day 3 bleomycin treated SFTPC$\mathrm{Cre}$;Has $2^{\text {flox/flox }}$ mice was lower compared to that of AEC2s from control mice (Fig. 4d). CD24-Sca-1- AEC2s from bleomycin injured SFTPC-Cre;Has $2^{\text {flox/flox }}$ mice showed markedly lower CFEs relative to that of AEC2s from Has $2^{\text {flox/flox }}$ littermates (Fig. 4e). $\mathrm{EpCAM}^{+} \mathrm{Lin}^{-}$epithelial cells from bleomycin injured SFTPC-Cre;Has $2^{\text {flox/flox }}$ mice also showed more apoptosis compared to the cells from littermate controls (Supplementary Fig. 5e). Unlike AEC2s isolated from $T 1 \mathrm{r}^{-/}{ }^{-}$, AEC2s from bleomycin-injured SFTPCCre;Has $2^{\text {flox/flox }}$ mice responded to Healon treatment giving rise to robust colony formation relative to the Healon-free Matrigel medium mix (Fig. 4f).

We hypothesized that lower cell surface HA on AEC2s would lead to enhanced susceptibility to bleomycin injury. Indeed, SFTPC-Cre;Has $2^{\text {flox/flox }}$ mice showed less 
survival (Fig. 4g) and more severe lung fibrosis at day 21 after bleomycin treatment as compared to injured littermate controls as assessed by hydroxyproline content (Fig. 4h) and trichrome staining (Fig. 4i).

\section{IL-6 promotes AEC2 renewal}

Our previous observation that HA-TLR4 interactions were associated with constitutive NF$\mathrm{\kappa B}$ activation within AEC2 cells ${ }^{11}$ led us to search for factors downstream of this signaling pathway that might mediate the protective effects of HA and TLR4 on AEC2s. We evaluated BALF composition at various times after injury and found that IL-6 was lower in the BALF of both $\mathrm{Tlr}^{-/-}$and SFTPC-Cre;Has $2^{\text {flox/flox }}$ mice compared to their corresponding controls (Fig. 5a,b). Microarray analysis also showed that 116 expression was among a cluster of genes that were down-regulated in $T 1 \mathrm{r}^{-/-} \mathrm{CD} 24^{-} \mathrm{Sca}-1^{-}$AEC2s compared to WT (Supplementary Fig. 6a). RT-PCR analysis confirmed that Il6 expression was lower in TLR4 deficient CD24-Sca-1- AEC2s than that of WT AEC2s 4 days after bleomycin (Supplementary Fig. 6b).

To test the hypothesis that the impaired production of IL- 6 might contribute to the impaired

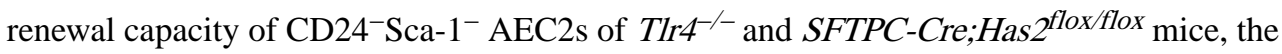
CFEs of CD24-Sca-1- AEC2s in 3-D organoid culture following treatment with either recombinant IL-6 protein or anti-IL-6 antibodies were evaluated. Anti-IL-6 antibodies reduced, while IL-6 protein increased, CFEs of CD24-Sca-1- AEC2s from bleomycin treated WT mice in a dose dependent manner (Fig. 5c,d). STAT3 inhibition abolished the effect of IL6-promoted colony formation of CD24-Sca-1- AEC2s (Supplementary Fig. 6c), suggesting IL-6 promotes AEC2 renewal through STAT3. In addition, IL-6 protein promoted CFEs of CD24-Sca-1- AEC2s from both bleomycin treated $T / r 4^{-/-}$and SFTPCCre;Has $2^{\text {flox/flox }}$ mice (Fig. 5e,f).

To determine if IL-6 regulates the fibrotic phenotype in vivo, we delivered IL-6 protein and anti-IL-6 antibodies to bleomycin-treated mice at the early stage of lung injury. IL-6 treatment of $\mathrm{TIr}_{4^{-/}}$mice resulted in more CD24-Sca-1- AEC2s recovery, greater Ki67 staining of CD24-Sca- $1^{-}$AEC2s, and lower BALF total proteins, compared to $T 1 \mathrm{r}^{-/-}$mice treated with control buffer (Fig. 5g). IL-6 treatment of $\mathrm{Tlr}^{4^{-/}}$mice did not impact AEC2 cell apoptosis (Supplementary Fig. 6d) and inflammatory cell infiltration compared to that of buffer-treated controls (Supplementary Fig. 6e). Bleomycin-injured TIr $4^{-/-}$mice treated with IL-6 protein showed an increased survival compared to buffer treatment (Supplementary Fig. 6f). In addition, hydroxyproline content was lower in the lungs of $\mathrm{TIr}^{4^{-/}}$mice treated with bleomycin and IL-6 protein compared with bleomycin alone (Fig. 5h). Similarly, IL-6 treatment resulted in lower hydroxyproline content in the lungs of SFTPC-Cre;Has $2^{\text {flox/flox }}$ mice injured with bleomycin compared to those treated with buffer (Fig. 5i). Conversely, bleomycin-injured WT mice treated with anti-IL-6 antibodies showed greater mortality compared to the mice treated with control IgG (Supplementary Fig. 6g), but we did not observe lower hydroxyproline levels (data not shown). Further, delivery of IL-6 protein to WT mice did not alter survival or hydroxyproline content compared to that of the mice treated with bleomycin alone (data not shown). 


\section{AEC2s from IPF patients have lower cell surface HA expression}

In order to determine if our observations in mouse model of Has2-deficient AEC2s have relevance to human disease, we isolated AEC2s from lung explants of human subjects who have undergone lung transplantation for IPF. Using cell surface markers ${ }^{29}$, we were able to sort AEC2s as $\mathrm{CD}^{2} 1^{-} \mathrm{CD} 45^{-} \mathrm{EpCAM}^{+} \mathrm{HTII}-280^{+}$cells from total single cell suspensions of lung tissues (R3, Supplementary Fig. 7a). We observed a dramatically lower percentage of EpCAM ${ }^{+} \mathrm{HTII}-280^{+} \mathrm{AEC} 2 \mathrm{~s}$ within the gated CD31-CD45- $\left(\mathrm{Lin}^{-}\right)$cells in the cell suspension of lung tissue from patients with IPF relative to those from healthy donors (Fig. $6 \mathrm{a}, \mathrm{b})$. Cell surface HA was markedly diminished on HTII- $280^{+}$AEC2s from explant lung tissues of the individuals with IPF as compared to those from healthy donors, similar to what we observed in our mouse model (Fig. 6c,d). Lower HAS2 expression in HTII-280 ${ }^{+}$AEC2s from the IPF patients relative to that of the cells from healthy donors was detected by q-PCR (Fig. 6e). These data suggest that our mouse model of targeted deletion of Has 2 in distal lung epithelium recapitulates key aspects of severe pulmonary fibrosis in human disease.

Human HTII-280 ${ }^{+}$AEC2s expressed TLR4 (Supplementary Fig. 7b), but minimal TLR2 (Supplementary Fig. 7c), consistent with a previous report of TLR4 and TLR2 expression on human alveolar epithelial cells ${ }^{30}$. However, there was no difference in TLR4 expression on HTII-280 ${ }^{+}$AEC2s between subjects with IPF and healthy donors (Supplementary Fig. 7b).

\section{AEC2s from IPF patients have impaired colony-forming capacity}

The observation that HTII- $280^{+}$AEC2s from lung tissue with severe pulmonary fibrosis have lower expression of cell surface HA led us to investigate the renewal capacity of these cells. We assessed the renewal capacity of human HTII-280 AEC2s using 3D matrigel culture (Supplemental Fig. 7d). Similar to what we observed in murine AEC2s devoid of cell surface HA, HTII-280 ${ }^{+}$AEC2s from lung tissues of individuals with IPF had lower CFEs (Fig. 6f) and formed smaller colonies (Fig. 6g) relative to AEC2s from tissue from healthy donors. Furthermore, IL-6 protein markedly increased CFEs of the HTII-280 ${ }^{+}$AEC2s sorted from diseased lung compared to growth in medium without exogenous IL-6 (Fig. 6h). The concentration $(200 \mu \mathrm{g} / \mathrm{ml})$ of exogenous HA (Healon) that was effective in the murine model, increased colony formation of HTII- $280^{+}$AEC2s from healthy lungs but not AEC2s from the patients with IPF (Supplementary Fig. 7e). Higher concentrations of Healon were required to augment colony formation of HTII- $280^{+}$AEC2s from IPF lungs (Supplementary Fig. 7f).

To further investigate if loss of cell surface HA on HTII- $280^{+}$AEC 2 cells in the lungs from the patients with IPF contributed to the impaired renewal capacity of the cells, we flow sorted high HA expressing (HA ${ }^{\text {hi }}$ ) and lower HA expressing $\left(\mathrm{HA}^{\mathrm{lo}}\right) \mathrm{HTII}-280^{+}$AEC2 cells from lungs of both patients with IPF and healthy donors. The majority of HTII- $280^{+}$AEC2 cells in healthy lung were $\mathrm{HA}^{\text {hi }}$ cells, and in contrast, only a small portion of HTII- $280^{+}$ AEC 2 cells in the diseased lung were HA ${ }^{\text {hi }}$ cells (Fig. 6i). HA ${ }^{\text {lo }}$ HTII-280 $2{ }^{+}$AEC2 cells had lower colony formation efficiency compared to HA ${ }^{\text {hi }} \mathrm{HTII}-280^{+}$AEC2 cells either from diseased or healthy lungs (Fig. 6j). These data indicate that loss of cell surface HA on AEC2 cells directly contributes to the impaired renewal capacity. However, the $\mathrm{HA}^{\text {hi }} \mathrm{HTII}-280^{+}$ AEC2 cells from diseased lung still showed lower CFEs relative to HA ${ }^{\text {hi }} \mathrm{HTII}-280^{+}$AEC2 
cells from healthy lungs (Fig. 6j), suggesting there may be other factors contributing to impaired AEC2 renewal in IPF.

As IPF is a patchy disease, we tested if AEC2s from severely fibrotic areas behaved differently from AEC2s from portions of the lung with less fibrosis in the same lung explant from patients with IPF. Flow cytometry showed that cell suspensions from less fibrotic areas contained relatively higher numbers of HTII $-280^{+}$AEC2s compared to less severely fibrotic areas within the same lung (Supplementary Fig. 8a). However, the recovery of HTII-280 AEC2 from the less fibrotic areas was still markedly lower than the recovery from healthy donor lung (Fig. 6a and Supplementary Fig. 8a). CFE of HTII-280+ AEC2s was equally impaired when isolated from severely or less severely fibrotic regions (Supplementary Fig. 8b). Cell surface HA expression on HTII- $280^{+}$AEC2s was reduced to the same extent regardless of the degree of tissue fibrosis, although there was variation (Supplementary Fig. 8c).

\section{DISCUSSION}

One of the critical issues in the pathobiology of lung fibrosis is the insufficient understanding of molecular mechanisms that regulate AEC2 renewal both during tissue homeostasis and after injury. A major goal of this study was to dissect the role of innate immune receptors and endogenous extracellular matrix molecules in regulating AEC2 renewal and lung fibrosis. The main finding in this study is that the endogenous matrix glycosaminoglycan HA and the innate immune receptor TLR4 are required for optimal AEC2 renewal and limiting fibrosis after lung injury. Most importantly, we discovered a loss of cell surface HA of AEC2s from lungs with IPF and that directly contribute to the impaired renewal capacity of such AEC2s.

Previous work from our laboratory demonstrated that HA and TLR4 and TLR2 on epithelial cells were necessary to sustain basal NF- $\kappa B$ activation and prevent epithelial cell apoptosis ${ }^{11}$. In this study, we tested the hypothesis that cell surface HA and TLR4 are crucial for AEC2 renewal following injury. We show that AEC2s with deletion of either TLR4 or HAS2 have lower self-renewal capacity in vitro and lead to greater bleomycininduced lung injury in vivo. Exogenous HA promoted WT AEC2-mediated organoid formation but not with AEC2s from $T I r 4^{-/}$mice, suggesting the interaction between cell surface HA and TLR4 is essential for promoting AEC2 renewal. Further study is needed to determine if there are direct interactions between HA and TLR4.

Epithelial cells from multiple organs, including alveolar epithelial cells ${ }^{30}$, intestinal epithelial cells ${ }^{31,32}$, and keratinocytes ${ }^{33}$ express innate immune receptors such as TLR4. TLRs play a role not only in immunological responses, but also in epithelial protection during homeostasis and after injury. The intestinal epithelial TLR4 and commensal bacteria play a key role in protection against bacterial infection ${ }^{1,2}$. We provide evidence here that distal alveolar epithelial TLR4 and HA are crucial in protecting AEC2s from injury and promoting renewal. These data suggest that cell surface HA may function in a manner similar to commensal bacteria since the distal alveolar space is devoid of bacteria. 
Many downstream signaling events may be altered in the absence of HA-TLR interactions. We present evidence that insufficient production of IL-6 resulted from HA-TLR4 deficiency impacted the biology of lung repair both in vitro and in vivo. AEC2s isolated from both injured $\mathrm{Tlr}^{-/-}$and SFTPC-Cre;Has $2^{\text {flox/flox }}$ mice demonstrated impaired renewal capacity and this was significantly reversed by IL- 6 treatment. In addition, exogenous IL- 6 treatment during the period of AEC2 vulnerability enhanced AEC2 renewal and partially reverse the fibrotic phenotype of $T I r 4^{-/-}$and SFTPC-Cre;Has $2^{\text {flox/flox }}$ mice in vivo. IL-6 is produced by a variety of cell types including epithelial cells and macrophages ${ }^{34}$. The role of IL-6 in lung injury may be multifold. First, IL-6 may be produced in the reparative niche to promote stem cell renewal ${ }^{35-37}$. Secondly, IL-6 is part of a cytokine network that modulates inflammatory responses after bleomycin ${ }^{38}$. Moreover, fibroblasts produce IL-6 and IL-6/STAT3 signaling regulates lung fibrosis ${ }^{38,39}$. It is conceivable that IL-6 has distinct effects on the biology of fibrosis at varying stages of the disease process. Other factors including CXCL12 and fibroblast growth factor 2 (FGF2) may regulate AEC2 repair after lung injury. Plateletderived CXCL12 ${ }^{40}$, endothelial CXCR $7^{41}$, and epithelial FGF2 ${ }^{42}$ have been suggested in initiating alveolar repair. Further investigation into the role of HA-TLR4 signaling in regulating these mediators is warranted.

We targeted Has2 in SFTPC-positive cells throughout development. This population will include a variety of progenitor/stem cell populations that have recently been identified through lineage tracing $6,7,24,43,44$, or by single-cell transcriptomics analysis ${ }^{45}$. These would not include the recently reported lineage- negative population in the context of severe lung injury ${ }^{46}$. We cannot make conclusions regarding the precise stem cell/progenitor population in the adult mouse lung responsible for the observed phenotype of SFTPC-Cre;Has 2flox/flox mice. We believe that the loss of cell surface HA may be more relevant than the specific population. Future studies will be directed at deleting Has 2 in adult populations of progenitor cells.

A provocative finding in this study is that cell surface HA is significantly lower on AEC2s from patients with IPF compared to those from healthy individuals. In contrast, we did not identify significant differences in cell surface TLR4 expression between AEC2s from patients with IPF and healthy donors. One of the reasons for the loss of AEC2 surface HA may be a result of the deficiency in $H A S 2$ gene expression. AEC2 cells with low HA expression either from lungs of patients with IPF or healthy individuals had dramatically lower organoid forming ability suggesting a direct link between the loss of cell surface HA and impaired renewal capacity of AEC2s. The finding that exogenous HA was able to augment colony forming capacity of AEC2s from fibrotic lungs further supports the concept that the loss of cell surface HA is a cause of impaired renewal of diseased AEC2s. Higher concentrations of HA were required in human AEC2s, suggesting that there are differences with the murine system.

These findings support the concept that IPF is primarily a disease of AEC2 stem cell failure. We have identified the loss of the HA synthesizing enzyme in AEC2s as a primary defect leading to the susceptibility to fibrosis. In addition, we have shown that innate immune receptors can promote epithelial regeneration in addition to the established functions in inflammation. Our mouse model of targeted deletion of Has 2 in distal lung epithelium 
recapitulates key aspects of severe pulmonary fibrosis in human disease. Importantly, AEC2 cells isolated from lung explants of patients with chronic obstructive pulmonary disease (COPD), another severe lung disease but with a very different pathology expressed cell surface HA levels similar to AEC2s from healthy donors (data not shown), suggesting that loss of cell surface HA on AEC2s is a unique feature of severe pulmonary fibrosis. Future studies will be directed toward ascertaining the mechanisms by which the silencing of HAS2 and subsequent loss of cell surface HA promotes a pro-fibrotic milieu, and to identify approaches to restoring cell surface HA on epithelial cells as well as epithelial cell integrity. These endeavors could lead to new therapeutic approaches in severe pulmonary fibrosis.

\section{METHODS}

Methods and any associated references are available in the online version of the paper.

\section{Supplementary Material}

Refer to Web version on PubMed Central for supplementary material.

\section{Acknowledgments}

We thank Maura Leonado, Yoosun Jung, and Vivian Hu for their technical support. This work was supported by NIH grants P01 HL108793, R01 HL060539, HL077291, and AI052201 (P.W.N.), and R01 HL122068 (D.J.), and a grant from California Institute for Regenerative Medicine (RB5-07302 to P.W.N). We appreciate B.L.M. Hogan for critical reading of the manuscript and invaluable comments. We are grateful to S. Akira (University of Osaka, Japan), Y. Yamaguchi (Sanford-Burnham Medical Research Institute), and L. Dobbs (UCSF) for providing critical reagents for the study.

\section{References}

1. Rakoff-Nahoum S, Hao L, Medzhitov R. Role of toll-like receptors in spontaneous commensaldependent colitis. Immunity. 2006; 25:319-329. [PubMed: 16879997]

2. Rakoff-Nahoum S, Paglino J, Eslami-Varzaneh F, Edberg S, Medzhitov R. Recognition of commensal microflora by toll-like receptors is required for intestinal homeostasis. Cell. 2004; 118:229-241. [PubMed: 15260992]

3. Fukata M, et al. Cox-2 is regulated by Toll-like receptor-4 (TLR4) signaling: Role in proliferation and apoptosis in the intestine. Gastroenterology. 2006; 131:862-877. [PubMed: 16952555]

4. Hogan BL, et al. Repair and regeneration of the respiratory system: complexity, plasticity, and mechanisms of lung stem cell function. Cell Stem Cell. 2014; 15:123-138. [PubMed: 25105578]

5. Kotton DN, Morrisey EE. Lung regeneration: mechanisms, applications and emerging stem cell populations. Nat Med. 2014; 20:822-832. [PubMed: 25100528]

6. Desai TJ, Brownfield DG, Krasnow MA. Alveolar progenitor and stem cells in lung development, renewal and cancer. Nature. 2014; 507:190-194. [PubMed: 24499815]

7. Barkauskas CE, et al. Type 2 alveolar cells are stem cells in adult lung. J Clin Invest. 2013; 123:3025-3036. [PubMed: 23921127]

8. Hofer CC, Woods PS, Davis IC. Infection of mice with influenza A/WSN/33 (H1N1) virus alters alveolar type II cell phenotype. Am J Physiol Lung Cell Mol Physiol. 2015; 308:L628-638. [PubMed: 25595651]

9. Liu Y, Suresh Kumar V, Zhang W, Rehman J, Malik AB. Activation of Type II Cells Into Regenerative Sca-1 Cells During Alveolar Repair. Am J Respir Cell Mol Biol. 2014; 53:113-124.

10. Rock JR, et al. Multiple stromal populations contribute to pulmonary fibrosis without evidence for epithelial to mesenchymal transition. Proc Natl Acad Sci U S A. 2011; 108:E1475-1483.

[PubMed: 22123957] 
11. Jiang D, et al. Regulation of lung injury and repair by Toll-like receptors and hyaluronan. Nat Med. 2005; 11:1173-1179. [PubMed: 16244651]

12. Jiang D, Liang J, Li Y, Noble PW. The role of Toll-like receptors in non-infectious lung injury. Cell Res. 2006; 16:693-701. [PubMed: 16894359]

13. Jiang D, Liang J, Noble PW. Hyaluronan in tissue injury and repair. Annu Rev Cell Dev Biol. 2007; 23:435-461. [PubMed: 17506690]

14. Noble PW, Jiang D. Matrix regulation of lung injury, inflammation, and repair: the role of innate immunity. Proc Am Thorac Soc. 2006; 3:401-404. [PubMed: 16799081]

15. Jiang D, Liang J, Noble P. Hyaluronan as an Immune Regulator in Human Diseases. Physiol Rev. 2011; 91:221-264. [PubMed: 21248167]

16. Camenisch TD, et al. Disruption of hyaluronan synthase-2 abrogates normal cardiac morphogenesis and hyaluronan-mediated transformation of epithelium to mesenchyme. J Clin Invest. 2000; 106:349-360. [PubMed: 10930438]

17. ATS/ERS. Idiopathic pulmonary fibrosis: diagnosis and treatment. International consensus statement. The joint statement of American Thoracic Society (ATS), and the European Respiratory Society (ERS). Am J Respir Crit Care Med. 2000; 161:646-664. [PubMed: 10673212]

18. Selman M, et al. Idiopathic pulmonary fibrosis: pathogenesis and therapeutic approaches. Drugs. 2004; 64:405-430. [PubMed: 14969575]

19. Noble PW, Barkauskas CE, Jiang D. Pulmonary fibrosis: patterns and perpetrators. J Clin Invest. 2012; 122:2756-2762. [PubMed: 22850886]

20. Amin RS, et al. Surfactant protein deficiency in familial interstitial lung disease. J Pediatr. 2001; 139:85-92. [PubMed: 11445799]

21. Thomas AQ, et al. Heterozygosity for a surfactant protein $\mathrm{C}$ gene mutation associated with usual interstitial pneumonitis and cellular nonspecific interstitial pneumonitis in one kindred. Am J Respir Crit Care Med. 2002; 165:1322-1328. [PubMed: 11991887]

22. Garcia CK. Idiopathic pulmonary fibrosis: update on genetic discoveries. Proc Am Thorac Soc. 2011; 8:158-162. [PubMed: 21543794]

23. Alder JK, et al. Telomere dysfunction causes alveolar stem cell failure. Proc Natl Acad Sci U S A. 2015; 112:5099-5104. [PubMed: 25840590]

24. Chen $\mathrm{H}$, et al. Airway epithelial progenitors are region specific and show differential responses to bleomycin-induced lung injury. Stem Cells. 2012; 30:1948-1960. [PubMed: 22696116]

25. Mummert ME, Mohamadzadeh M, Mummert DI, Mizumoto N, Takashima A. Development of a peptide inhibitor of hyaluronan-mediated leukocyte trafficking. J Exp Med. 2000; 192:769-779. [PubMed: 10993908]

26. Li Y, et al. Severe lung fibrosis requires an invasive fibroblast phenotype regulated by hyaluronan and CD44. J Exp Med. 2011; 208:1459-1471. [PubMed: 21708929]

27. Matsumoto K, et al. Conditional inactivation of Has2 reveals a crucial role for hyaluronan in skeletal growth, patterning, chondrocyte maturation and joint formation in the developing limb. Development. 2009; 136:2825-2835. [PubMed: 19633173]

28. Eblaghie MC, Reedy M, Oliver T, Mishina Y, Hogan BL. Evidence that autocrine signaling through Bmprla regulates the proliferation, survival and morphogenetic behavior of distal lung epithelial cells. Dev Biol. 2006; 291:67-82. [PubMed: 16414041]

29. Gonzalez RF, Allen L, Gonzales L, Ballard PL, Dobbs LG. HTII-280, a biomarker specific to the apical plasma membrane of human lung alveolar type II cells. J Histochem Cytochem. 2010; 58:891-901. [PubMed: 20566753]

30. Armstrong L, et al. Expression of functional toll-like receptor-2 and -4 on alveolar epithelial cells. Am J Respir Cell Mol Biol. 2004; 31:241-245. [PubMed: 15044215]

31. Abreu MT, et al. Decreased expression of Toll-like receptor-4 and MD-2 correlates with intestinal epithelial cell protection against dysregulated proinflammatory gene expression in response to bacterial lipopolysaccharide. J Immunol. 2001; 167:1609-1616. [PubMed: 11466383]

32. Neal MD, et al. Toll-like receptor 4 is expressed on intestinal stem cells and regulates their proliferation and apoptosis via the p53 up-regulated modulator of apoptosis. J Biol Chem. 2012; 287:37296-37308. [PubMed: 22955282] 
33. Pivarcsi A, et al. Expression and function of Toll-like receptors 2 and 4 in human keratinocytes. Int Immunol. 2003; 15:721-730. [PubMed: 12750356]

34. Oh K, et al. Epithelial transglutaminase 2 is needed for $\mathrm{T}$ cell interleukin-17 production and subsequent pulmonary inflammation and fibrosis in bleomycin-treated mice. J Exp Med. 2011; 208:1707-1719. [PubMed: 21746810]

35. Tadokoro T, et al. IL-6/STAT3 promotes regeneration of airway ciliated cells from basal stem cells. Proc Natl Acad Sci U S A. 2014; 111:E3641-3649. [PubMed: 25136113]

36. Tebbutt NC, et al. Reciprocal regulation of gastrointestinal homeostasis by SHP2 and STATmediated trefoil gene activation in gp130 mutant mice. Nat Med. 2002; 8:1089-1097. [PubMed: 12219085]

37. Zhang S, et al. Interleukin 6 mediates the therapeutic effects of adipose-derived stromal/stem cells in lipopolysaccharide-induced acute lung injury. Stem Cells. 2014; 32:1616-1628. [PubMed: 24449042]

38. Le TT, et al. Blockade of IL-6 Trans signaling attenuates pulmonary fibrosis. J Immunol. 2014; 193:3755-3768. [PubMed: 25172494]

39. O'Donoghue RJ, et al. Genetic partitioning of interleukin-6 signalling in mice dissociates Stat3 from Smad3-mediated lung fibrosis. EMBO Mol Med. 2012; 4:939-951. [PubMed: 22684844]

40. Rafii S, et al. Platelet-derived SDF-1 primes the pulmonary capillary vascular niche to drive lung alveolar regeneration. Nat Cell Biol. 2015; 17:123-136. [PubMed: 25621952]

41. Cao Z, et al. Targeting of the pulmonary capillary vascular niche promotes lung alveolar repair and ameliorates fibrosis. Nat Med. 2016; 22:154-162. [PubMed: 26779814]

42. Guzy RD, Stoilov I, Elton TJ, Mecham RP, Ornitz DM. Fibroblast growth factor 2 is required for epithelial recovery, but not for pulmonary fibrosis, in response to bleomycin. Am J Respir Cell Mol Biol. 2015; 52:116-128. [PubMed: 24988442]

43. Chapman HA, et al. Integrin alpha6beta4 identifies an adult distal lung epithelial population with regenerative potential in mice. J Clin Invest. 2011; 121:2855-2862. [PubMed: 21701069]

44. Lee JH, et al. Lung stem cell differentiation in mice directed by endothelial cells via a BMP4NFATc1-thrombospondin-1 axis. Cell. 2014; 156:440-455. [PubMed: 24485453]

45. Treutlein B, et al. Reconstructing lineage hierarchies of the distal lung epithelium using single-cell RNA-seq. Nature. 2014; 509:371-375. [PubMed: 24739965]

46. Vaughan AE, et al. Lineage-negative progenitors mobilize to regenerate lung epithelium after major injury. Nature. 2015; 517:621-625. [PubMed: 25533958] 
a

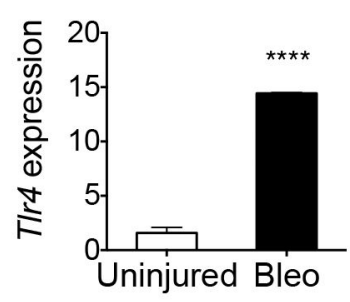

C

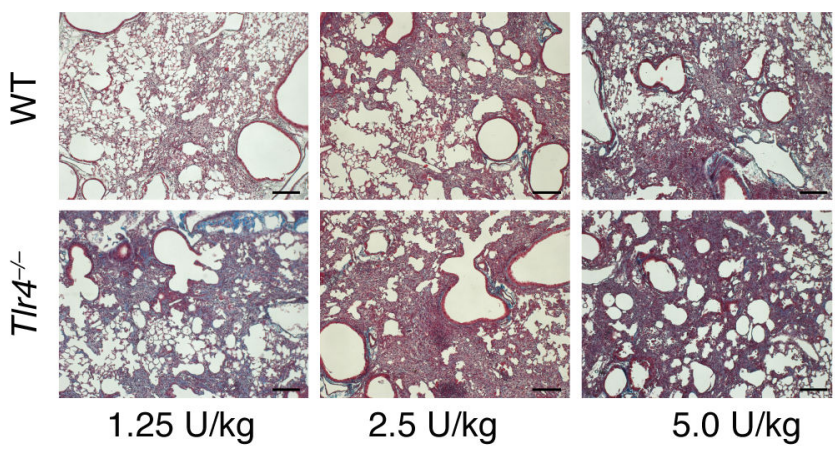

d

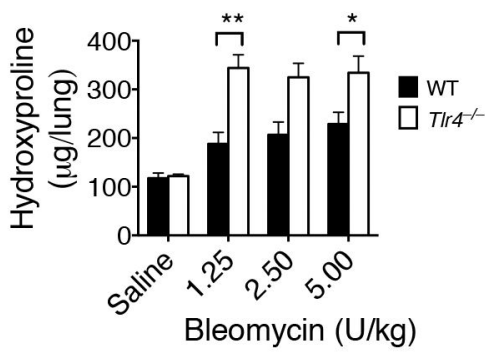

f

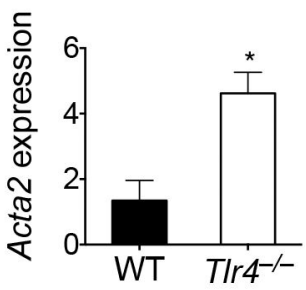

b

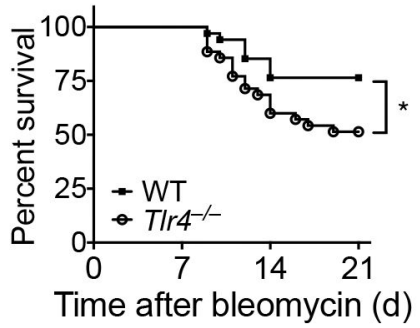

e

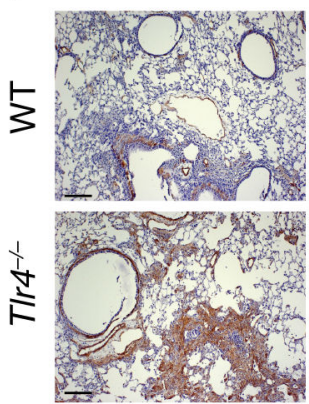

Figure 1.

$\mathrm{Tlr}^{{ }^{-/}}$mice demonstrate higher mortality and more severe fibrosis after bleomycin-induced lung injury. (a) TIr 4 expression in the lungs of untreated $(n=3)$ and bleomycin-treated wildtype (WT) mouse (day 7, $n=3$ ) as assessed by RT-PCR (****P<0.0001 by Student $t$-test). (b) Percentages of surviving Tlr $4^{-/-}$mice $(n=35)$ and WT mice $(n=34)$ plotted over a 21day period after intratracheal treatment with bleomycin $(2.5 \mathrm{U} / \mathrm{kg}) .{ }^{*} P<0.05$ by log-rank test. (c,d) Representative images (c) of trichrome staining and hydroxyproline contents (d) of lungs from $T l r 4^{-/}$and WT mice $21 \mathrm{~d}$ after bleomycin injury $(1.25-5 \mathrm{U} / \mathrm{kg})(\mathbf{d}$, for saline WT $n=5, T l r 4^{-/} n=4$; for $1.25 \mathrm{U}$, WT $n=6, T l r 4^{-/} n=9$; for $2.5 \mathrm{U}$, WT $n=4$, $T I r 4^{-/} n=4$; for $5 \mathrm{U}$, WT $n=9, T I r 4^{-/} n=12$; $* P<0.05$, ** $P<0.01$ by Two way analysis of variance (ANOVA) followed by Sidak's multiple comparison test). (e) Representative images of total 4 images photographed for each group of anti-a-SMA immunostaining of 
lungs from $T I \mathrm{r}^{-/-}$and WT mice 21 days after bleomycin injury (2.5 U/kg). (f) a-SMA (Acta2) expression in lungs from bleomycin-treated $T l r 4^{-/}(n=4)$ and WT mice $(n=4)$ as assessed by qPCR $(* P<0.05$ by Student $t$-test). Scale bars, $200 \mu \mathrm{m}$. 
a

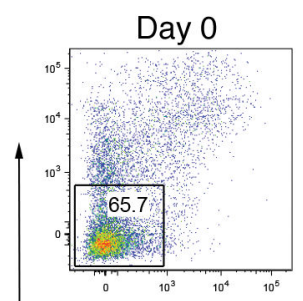

Day 7

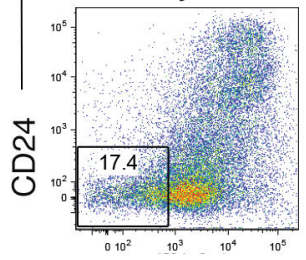

Sca-1

b

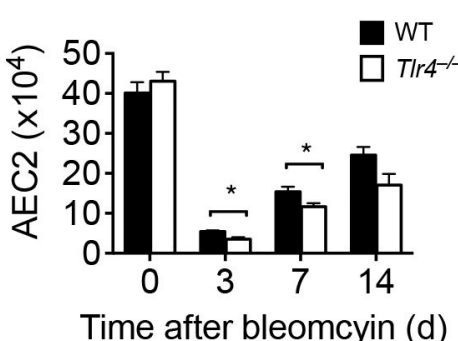

Day 1

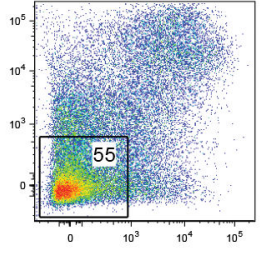

Day 14

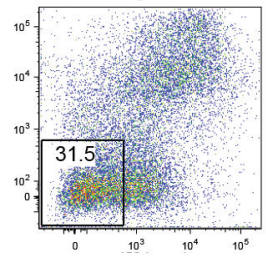

C

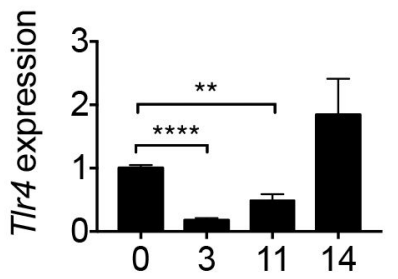

Time after bleomcyin (d)

d
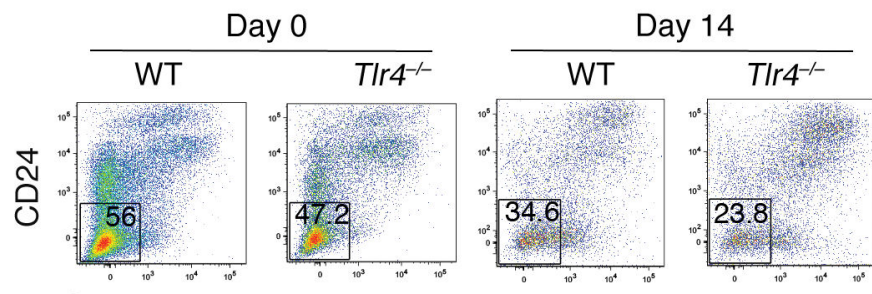

Sca-1

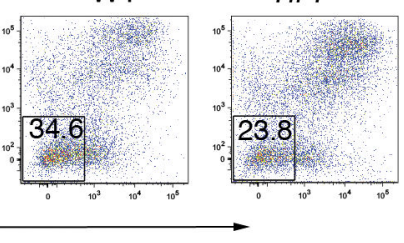

Figure 2.

$\mathrm{TIr}^{4^{-/}}$deficiency leads to loss of AEC2 cells in mouse lungs after bleomycin-induced injury. (a) Percent changes of CD24-Sca- $1^{-}$AEC2 population gated from total lung epithelial cells $\left(\mathrm{EpCAM}^{+} \mathrm{Lin}^{-}\right)$in bleomycin treated WT mice. (b) Numbers of CD24-Sca- $1^{-}$AEC2s recovered from the lungs of bleomycin treated $T I \mathrm{r}^{-/-}$and WT mice at indicated time points (for day $0, n=3$ each; for day $3, n=4$ each; for day 7, WT $n=6$, $T I r 4^{-/-} n=4$; for day $14, n=5$ each; $* P<0.05$ by Student $t$-test). (c) Tlr 4 expression of CD24-Sca-1- AEC2s flow-sorted from bleomycin treated WT mice at indicated time points by RT-PCR ( $n=4$ each group; $* * P<0.01 ; * * * * P<0.0001$ by one-way ANOVA followed by Sidak's multiple comparison test). (d) Gated CD24-Sca-1- AEC2s in $T 1 r 4^{-/-}$and WT mouse lungs day 0 and day 14 after bleomycin. 
a

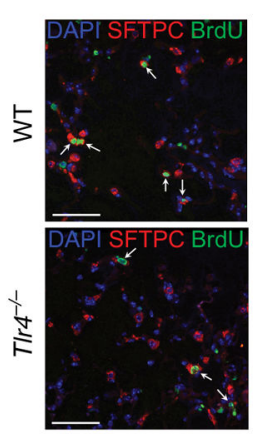

e

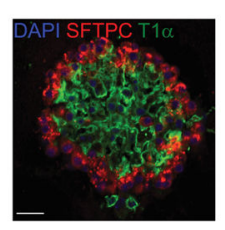

b

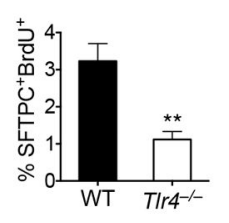

c

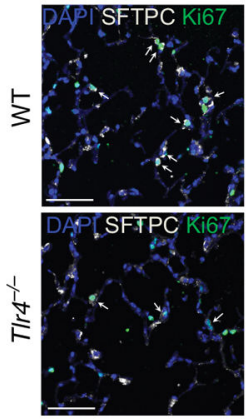

g

f

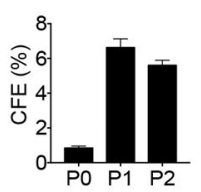

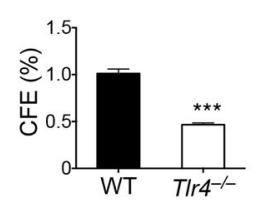

d

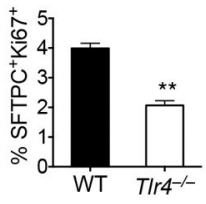

i

j
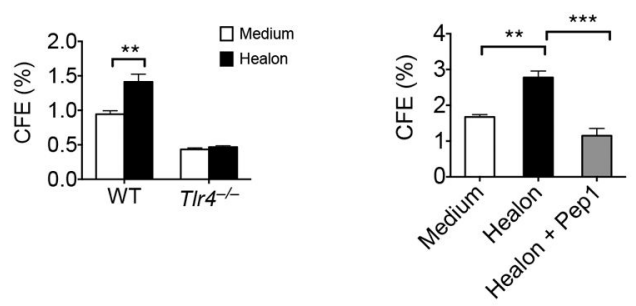

Figure 3.

AEC2 differentiation and proliferation requires TLR4 signaling. (a) Representative images of 9 images photographed for each group of co-staining of SFTPC and BrdU of lung sections from WT and $T l r 4^{-/}$mice after bleomycin. Arrows indicate overlap staining. (b) Percentage of SFTPC ${ }^{+} \mathrm{BrdU}^{+}$in total SFTPC ${ }^{+}$cells of WT $(n=6)$ and $T l r 4^{-/}$mouse lungs $(n=5)$. (c,d) Representative images of 9 images photographed for each group of co-staining (c) and percentage (d) of SFTPC ${ }^{+} \mathrm{Ki}^{+} 7^{+}$in total $\mathrm{SFTPC}^{+}$cells of lung sections of WT $(n=$ 3) and $T l r 4^{-/}$mice $(n=3)$ after bleomycin. (e) Representative image of total 6 colonies of WT AEC2s stained with SFTPC and T1a. (f) Replating CFEs of CD24-Sca- $1^{-}$AEC2s from bleomycin-treated WT mice. Passage (P) $0(n=8)$; P1 $(n=3)$; and P2 $(n=3)$. (g) CFEs of CD24-Sca-1- AEC2s of $T I r 4^{-/}(n=3)$ and WT mice $(n=3) 3 \mathrm{~d}$ after bleomycin. (h) Colony sizes of CD24-Sca- $1^{-}$AEC2s of TIr $4^{-1}(n=44)$ and WT mice $(n=39)$. (i) CFEs of CD24 ${ }^{-}$Sca-1- ${ }^{-}$AEC2s with and without Healon (WT $n=5$ each, $T I r 4^{-/} n=3$ each). (j) CFEs of uninjured WT AEC2s with medium only $(n=4)$, Healon $(n=5)$, or Healon and pep-1 $(n=3)$. Scale bars, $50 \mu \mathrm{m}(\mathbf{a}, \mathbf{c})$, and $20 \mu \mathrm{m}(\mathbf{e}) . * * P<0.01, * * * P<0.001, * * * * P<$ 0.0001 by Student $t$-test $(\mathbf{b}, \mathbf{d}, \mathbf{g})$, Mann-Whitney $U$-test (h), two-way ANOVA followed by Sidak's multiple comparison test (i), by one-way ANOVA followed by Sidak's multiple comparison test (j). 
a

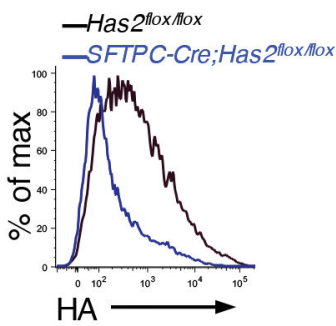

e

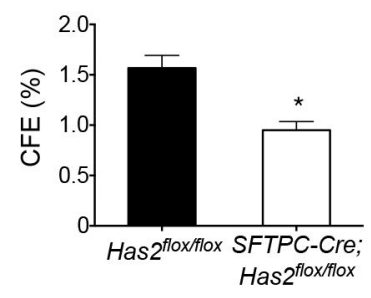

h

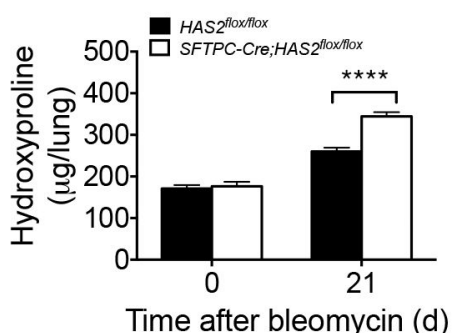

b

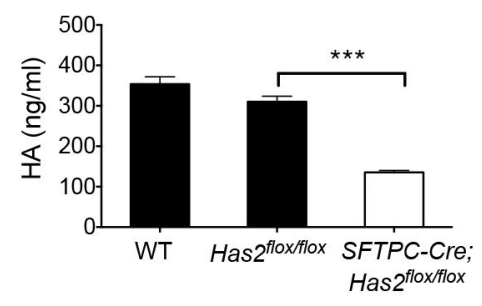

$\mathbf{f}$

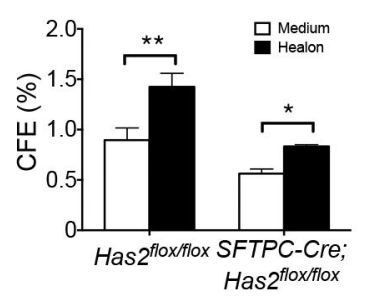

C

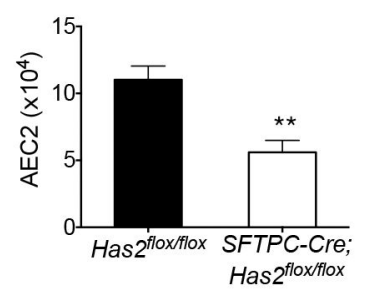

d

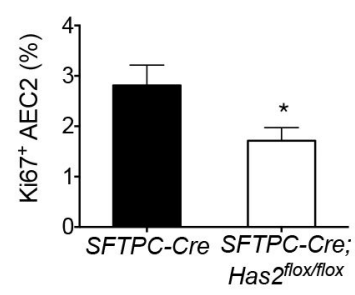

g

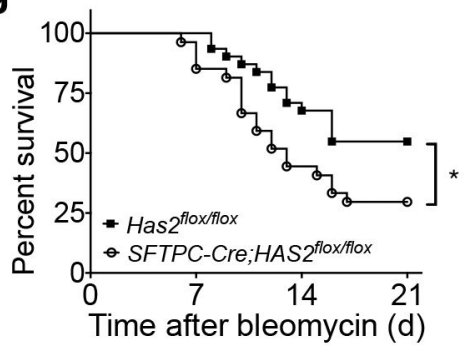

i

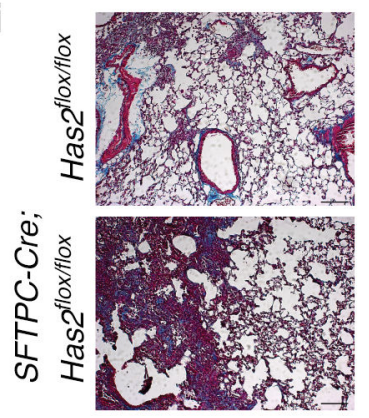

Figure 4.

Has 2 deficient AEC2s have lower colony forming capacity. (a) Cell surface HA of CD24-Sca-1- AEC2s of SFTPC-Cre;Has $2^{\text {flox/flox }}$ and Has $2^{\text {flox/flox }}$ mice. (b) HA concentration of CD24-Sca-1- AEC2s from SFTPC-Cre;Has $2^{\text {flox/flox }}(n=3)$, Has $2^{\text {flox/flox }}$ $(n=3)$, and WT mice $(n=3)$. (c) Numbers of CD24-Sca- $1^{-}$AEC2s recovered from bleomycin day 3 SFTPC-Cre;Has $2^{\text {flox/flox }}(n=4)$ and Has $2^{\text {flox } / f l o x}$ mice $(n=6)$. (d) Percent of Ki67 ${ }^{+}$AEC2s in total gated CD24-Sca-1- AEC2s from bleomycin-treated day 3 SFTPCCre;Has $2^{\text {flox/flox }}(n=9)$ and SFTPC-Cre mice $(n=9)$. (e) CFEs of CD24-Sca-1- AEC2s sorted from SFTPC-Cre;Has $2^{\text {flox/flox }}(n=3)$ and Has $2^{\text {flox/flox }}(n=3) 3$ days after bleomycin. (f) CFEs of CD24-Sca-1- AEC2s sorted from SFTPC-Cre;Has $2^{\text {flox/flox }(~} n=4$ each) and Has $2^{\text {flox/flox }}$ (medium $n=5$ and Healon $n=4$ ) 3 days after bleomycin. (g) Survival curves of SFTPC-Cre;Has $2^{\text {flox/flox }}(n=27)$ and Has $2^{\text {flox/flox }}$ littermates $(n=31)$ after bleomycin. (h) Hydroxyproline contents in lungs of SFTPC-Cre;Has $2^{\text {flox/flox }}$ and Has $2^{\text {flox/flox }}$ mice (for day 0 SFTPC-Cre;Has $2^{\text {flox/flox }} n=5$, Has2 $2^{\text {flox/flox }} n=7$; for day 21 SFTPC-Cre;Has $2^{\text {flox/flox }} n=$ 6. Has $2^{\text {flox } / f l o x} n=10$ ). (i) Representative images of 6 images photographed for each group of trichrome staining of lung sections of SFTPC-Cre;Has $2^{\text {flox/flox }}$ and Has $2^{\text {flox/flox }}$ day 21 after bleomycin. Scale bars, $200 \mu \mathrm{m}$. $* P<0.05$, $* * P<0.01$, *** $P<0.001$, **** $P<0.0001$ by one-way ANOVA followed by Sidak's multiple comparison test (b), two-way ANOVA 
followed by Holm-Sidak's (f) or Sidak's multiple comparison test (h), Student $t$-test (c,d,e), or by long-rank test (g). 
a

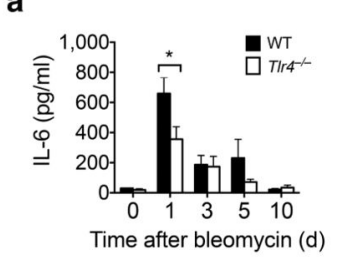

d

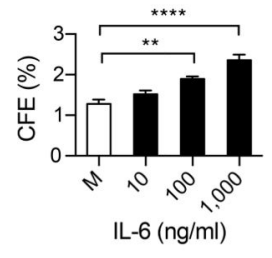

g
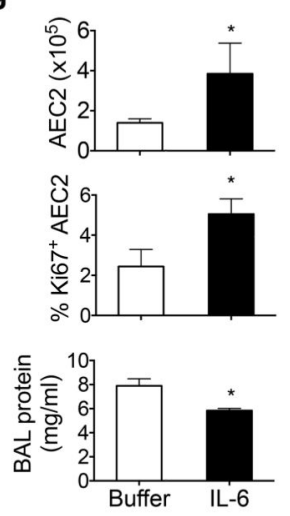

b

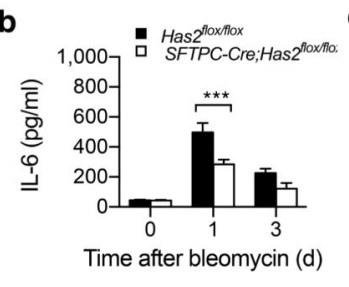

e

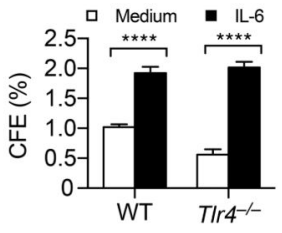

h

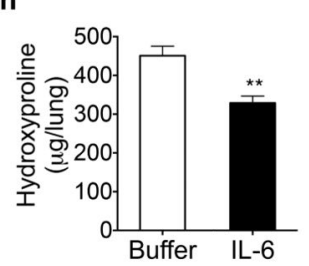

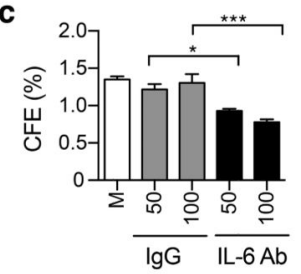

$f$

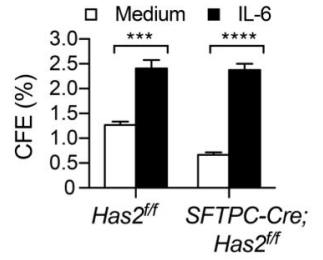

i

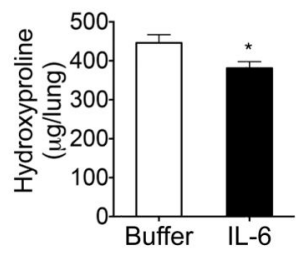

Figure 5.

IL-6 promotes AEC renewal and limits lung fibrosis. (a,b) IL-6 concentrations in BALF of $T / 4^{-1-}$ and WT mice (a) or SFTPC-Cre;Has $2^{\text {flox/flox }}$ and littermates (b) at indicated time points (in a, day 0, WT $n=3, T l r 4^{-/} n=5$; day $1, n=4$ each; day 3, WT $n=4, T l r 4^{-/} n=$ 6; day 5, WT $n=5, T l r 4^{-/} n=4$; day 10, WT $n=8, T l r 4^{-/} n=4$; in b, day $0, n=4$ each; day 1 , Has $2^{\text {flox/flox }} n=4$, SFTPC-Cre;Has $2^{\text {flox/flox }} n=5$; day 3 , Has $2^{\text {flox/flox }} n=4, S F T P C$ Cre;Has $2^{\text {flox/flox }} n=3$ ). (c,d) CFEs of CD24-Sca-1- AEC2s from bleomycin-treated WT mice were treated with (c) anti-IL-6 antibodies and control IgG $(\mu \mathrm{g} / \mathrm{ml})$ or $(\mathbf{d})$ IL-6 protein (in c, $n=3$ each; in d, $n=6$ except $10 \mathrm{ng} / \mathrm{ml}$ group where $n=3$ ). (e,f) CFEs of CD24-Sca-1 ${ }^{-}$AEC2s from bleomycin-treated (e) $T I r 4^{-/}$and WT mice or (f) SFTPCCre;Has $2^{\text {flox/flox }}$ and littermates with IL-6 protein (100 ng/ml) (in e, for medium, WT n = 6, $T I r 4^{-/} n=5$, for IL-6 $n=3$ each; in f, $n=3$ each). (g) Bleomycin-treated $T l 4^{-/}$mice with IL-6 protein or buffer. CD24-Sca-1- AEC2s recovered (top panel, buffer $n=8$, IL-6 $n=4$ ), Ki67 staining of AEC2s (middle panel, buffer $n=6$, IL-6 $n=8$ ), and total BAL protein (bottom panel, $n=4$ each). (h,i) Hydroxyproline contents of bleomycin-treated (h) Tlr $4^{-/}$ mice or (i) SFTPC-Cre;Has $2^{\text {flox/flox }}$ mice with IL-6 protein or buffer (in $\mathbf{h}$, buffer $n=4$, IL-6 $n=9$; in $\mathbf{i}$, buffer $n=9$, IL-6 $n=6)$. $* P<0.05, * * P<0.01, * * * P<0.001, * * * * P<0.0001$ by two-way ANOVA followed by Sidak's multiple comparison test (a,b,e,f), one-way ANOVA followed by Sidak's multiple comparison test $(\mathbf{c}, \mathbf{d})$, or by Student $t$-test $(\mathbf{g}, \mathbf{h}, \mathbf{i})$. 
a

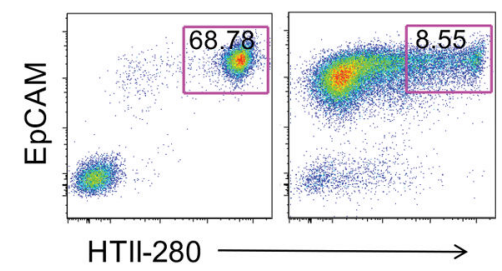

C

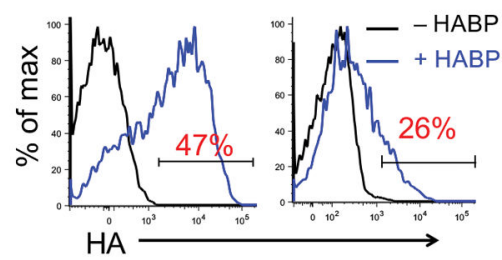

b

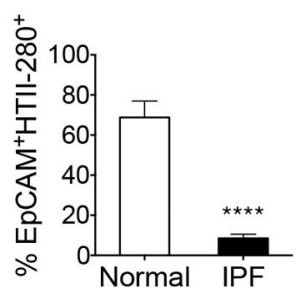

d

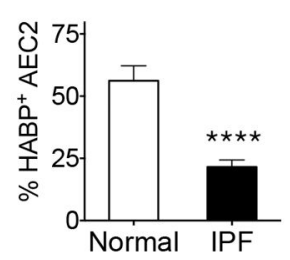

e

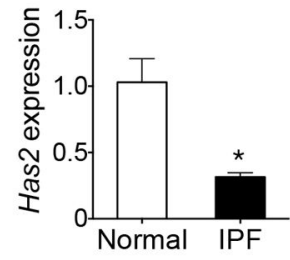

f

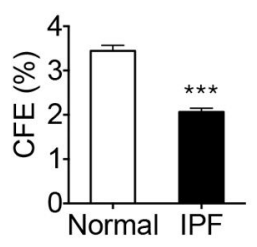

i

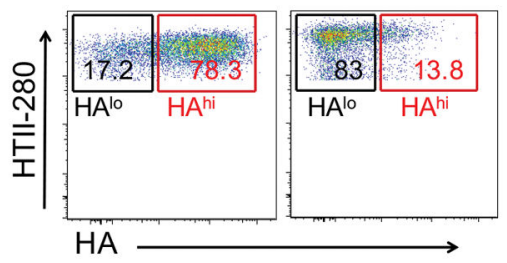

g

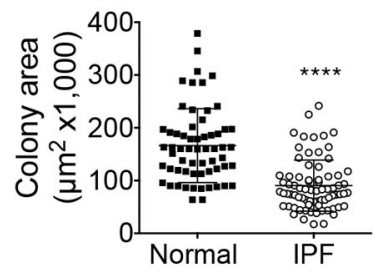

h

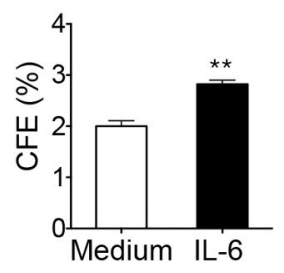

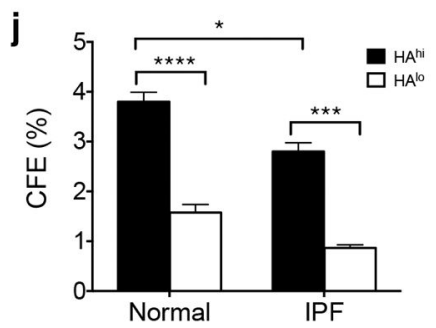

Figure 6.

Loss of cell surface HA and impaired renewal capacity of IPF AEC2s. (a) HTII-280 AEC2s in lungs from healthy donors (left) or from patients with IPF lungs (right). (b) Percentage of HTII-280 ${ }^{+}$AEC2s in lungs from healthy donors $(n=4)$ or patients with IPF $(n$ =7). (c) Cell surface HA on HTII-280 ${ }^{+}$AEC2s from healthy (left) or diseased lungs (right). (d) Percentages of $\mathrm{HABP}^{+} \mathrm{HTII}-280^{+}$AEC2s from healthy $(n=6)$ and diseased lungs $(n=$ 9). (e) Has 2 expression between HTII- $280^{+}$AEC 2 s from healthy and diseased lungs were determined using RT-PCR ( $n=3$ each). The results were repeated with AEC2s from two more subjects with IPF). (f) CFE between HTII-280 ${ }^{+}$AEC2s from healthy and diseaed lungs ( $n=3$ each). The results were repeated with AEC2s from three healthy controls and five more subjects with IPF. (g) Colony sizes of HTII-280 ${ }^{+}$AEC2s from lungs from healthy donors $(n=61)$ and patients with IPF $(n=75)$. (h) CFE of diseased HTII-280 ${ }^{+}$AEC2s treated with or without IL-6 $(n=3$ each). The results were repeated with AEC2s from two 
more individuals with IPF. (i) Flow gated $\mathrm{HA}^{\text {hi }}$ and $\mathrm{HA}^{\text {low }} \mathrm{HTII}-280^{+}$AEC2s from healthy donors (left) and patients with IPF (right). (j) CFE between HA ${ }^{\text {hi }}$ and HA ${ }^{\text {low }}$ AEC2s from lungs of healthy donors ( $n=4$ each) and patients with IPF ( $n=3$ each). The results were repeated with cells from two more subjects of each group. $* P<0.05, * * P<0.01, * * * P<$ $0.001, * * * * P<0.0001$ by Student $t$-test $(\mathbf{b}, \mathbf{d}, \mathbf{e}, \mathbf{f}, \mathbf{h})$, by Mann-Whitney $U$-test $(\mathbf{g})$, or by twoway ANOVA followed by Sidak's multiple comparison test (j). 